\title{
Sampling-Based Path Planning on Configuration-Space Costmaps
}

\author{
Léonard Jaillet, Juan Cortés, and Thierry Siméon
}

\begin{abstract}
This paper addresses path planning to consider a cost function defined over the configuration space. The proposed planner computes low-cost paths that follow valleys and saddle points of the configuration-space costmap. It combines the exploratory strength of the Rapidly exploring Random Tree (RRT) algorithm with transition tests used in stochastic optimization methods to accept or to reject new potential states. The planner is analyzed and shown to compute low-cost solutions with respect to a path-quality criterion based on the notion of mechanical work. A large set of experimental results is provided to demonstrate the effectiveness of the method. Current limitations and possible extensions are also discussed.
\end{abstract}

Index Terms-Costmap planning, path quality, sampling-based motion planning.

\section{INTRODUCTION}

$\mathbf{S}$ AMPLING-BASED path planning has proven to be an effective framework that is suitable for a large class of problems in domains, such as robotics, manufacturing, computer animation, and computational biology (see [1] and [2] for a survey). These techniques handle complex problems in high-dimensional spaces but usually operate in a binary world, which aims to find out collision-free solutions rather than the optimal path.

Specific path-planning methods have been developed in field robotics for outdoor navigation, where the goal is to find optimal paths according to a cost function, which is usually computed from a model of the terrain. Classical grid-based methods, such as $A^{*}$ or $D^{*}$ [3] can be used to compute resolution-optimal paths over a costmap. However, compared with sampling-based algorithms, these methods are limited to problems which involve low-dimensional spaces that can be discretized and searched using grid-search techniques.

Some recent works [4]-[8] have tried to bridge the gap between sampling-based planners and grid-based costmap planners. They mainly rely on the Rapidly exploring Random

Manuscript received November 16, 2009; revised March 10, 2010; accepted April 27, 2010. Date of publication June 3, 2010; date of current version August 10, 2010. This paper was recommended for publication by Associate Editor O. Brock and Editor K. Lynch upon evaluation of the reviewers' comments. This work was supported in part by the French National Research Agency under Project "GlucoDesign," in part by the European Community under FP7ICT Project 216239 "DEXMART," and in part by the Spanish Ministry of Science and Innovation under Project DPI2007-60858. The work of L. Jaillet was supported by Consejo Superior de Investigaciones Científicas under the JAE-Doc Fellowship.

L. Jaillet is with the Institut de Robòtica i Informàtica Industrial, Consejo Superior de Investigaciones Científicas, Universitat Politecnica de Catalunya, Barcelona 08028, Spain (e-mail: ljaillet@iri.upc.edu).

J. Cortés and T. Siméon are with CNRS; LAAS, F-31077 Toulouse, France, and also with Université de Toulouse; UPS, INSA, INP, ISAE; LAAS; F-31077 Toulouse, France (e-mail: jcortes@laas.fr; nic@laas.fr).

Color versions of one or more of the figures in this paper are available online at http://ieeexplore.ieee.org.

Digital Object Identifier 10.1109/TRO.2010.2049527

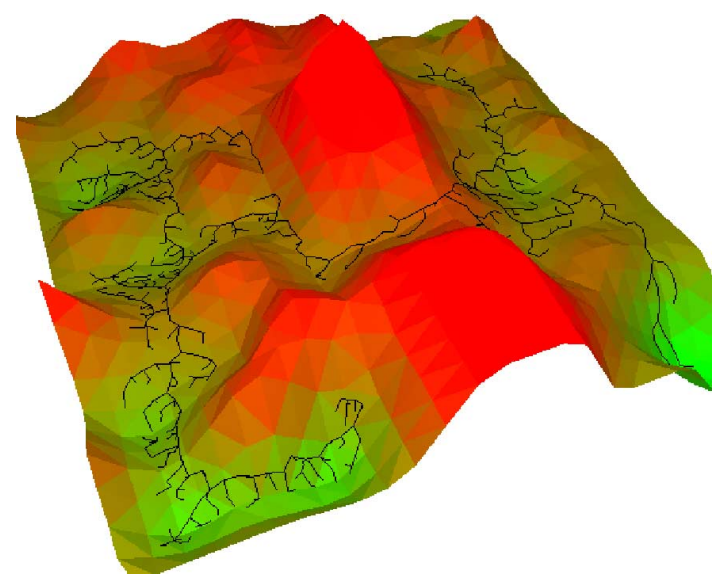

Fig. 1. Transition-based RRT on a 2-D costmap (the elevation corresponds to the costs). The exploration favors the expansion in valleys and saddle points, which connect to low-cost regions.

Tree (RRT) algorithm [9] and are generally focused on specific applications (e.g., real-time problems [7], [10] or statistical learning of feasible paths [8]) in the context of 2-D robot navigation problems.

This paper presents a general algorithm, called Transitionbased RRT (T-RRT), ${ }^{1}$ for path planning on configuration-space costmaps. The algorithm considers a user-given cost function defined over the configuration space as an additional input to the standard path-planning problem, and it produces solution paths that are not only feasible (e.g., collision free), but also have a good quality with respect to the input costmap. For instance, the costmap may correspond in outdoor navigation problems to the elevation map of the terrain in order to compute motions that minimize climbing of high-slope regions. In addition, in roboticmanipulation problems, the cost function may be defined from distances to be maximized between the robot and some objects, in order to find high-clearance solution paths. Finally, in computational biology applications, the costmap can be viewed as the energy landscape of the conformational space to be considered for the simulation of low-energy molecular motions.

The proposed algorithm combines the exploratory strength of RRTs with the efficiency of stochastic-optimization methods (e.g., Monte Carlo optimization and simulated annealing) that use transition tests to accept or reject new potential states. The filtering of the transition test relies on the gradient of cost function along the local motion to connect a given state to the RRT tree that results in an expansion biased to follow the valleys and the saddle points of the configuration-space costmap (see Fig. 1). Solution paths computed by T-RRT fulfill a quality

\footnotetext{
${ }^{1}$ The T-RRT planner was introduced in a shorter version published in [11]
} 
property based on the notion of mechanical work, which is also introduced in this paper as an effective criterion to evaluate path quality for costmap planning.

This paper is organized as follows. After a brief presentation of related work (see Section II), we introduce and discuss the notion of Minimal Work (MW) paths (see Section III). These paths are optimal according to a new criterion called mechanical work that is used to evaluate path quality. Comparison with other existing criteria shows the advantage of this criterion that may be more suitable in many situations, since it yields better paths following the low-cost valleys of the costmap. Additional properties of MW are also presented for a deep understanding of this notion. Section IV describes the T-RRT algorithm and explains the methods for self-tuning of parameters and for the expansion-rate control. Section V shows how T-RRT implicitly computes MW paths and discusses its probabilistic completeness. An experimental validation of the planner is conducted in Section VI. The overall efficacy of T-RRT is shown on different problems and positively compared with other existing techniques [4], [6]. Section VI also analyzes the influence of the intrinsic parameters of the algorithm on the overall performance, and results indicate that no specific tuning is actually needed. Section VII presents some extensions of T-RRT, and finally, conclusions are outlined in Section VIII.

\section{RELATED WORK}

Early potential field methods [12], as well as their combination with strategies to escape local minima, e.g., the randomized planner described in [13], rely on some numerical field defined over the configuration space that may be viewed as a specific kind of costmap. Note, however, that the artificial potential field of these methods is only defined as a way to plan collision-free paths, without considering path optimality. Thus, these methods do not address the problem considered here to compute lowcost, feasible paths from an arbitrarily complex costmap given as input to the planner.

Recent sampling-based planners have proven to be very effective to find feasible solutions that can be locally optimized in a postprocessing stage. Local path-optimization methods, such as the shortcut algorithm [14] are generally used to improve path quality with respect to simple criteria, e.g., path length, clearance, or a combination of both [15]. These smoothing methods only aim to locally improve a solution path, as opposed to the global exploration algorithm proposed in this paper. Moreover, their extension to arbitrary cost functions has not yet been addressed, and the resulting efficacy of such an extension remains to be further evaluated.

Only few papers consider sampling-based path planning on arbitrary cost spaces. An adaptation of the RRT-connect planner is used to find low-cost paths for rough terrain navigation in [4]. The idea is to keep new configurations only if their cost is under a given threshold, first initialized to a low value, and then iteratively increased during the search. One limitation of this technique comes from the nondecreasing threshold, which limits the efficiency of low-cost search to the vicinity of the root nodes. To overcome this issue, the extension proposed in [5] considers multiple RRTs grown from randomly sampled root configurations. However, this solution still expects an appropriate number of initial samples in order to get enough low-cost seeds among the space. Moreover, it requires a manual tuning of the parameter that controls the cost threshold-growth rate. This tuning is highly problem-dependent.

In [6], the heuristically guided RRT (hRRT) biases the search by using a quality measure based on the integral of the cost along the path from the root node and an estimation of the optimal cost to the goal. Such an approach, inspired from graph-search techniques, can also be found in the context of real-time applications [7], [10] and statistical learning of feasible paths [8]. However, with these techniques, the estimated cost to goal is heuristic, and tends to bias the search straight toward the goal at the expense of lower quality solution paths. Moreover, the aforementioned methods have only been demonstrated on simple low-dimensional examples with discrete cost states (invalid, low cost, and high cost, respectively). Their scalability and performance for problems which involve complex cost spaces in higher dimensions have yet to be established.

The T-RRT algorithm introduced in the following is inspired by Monte Carlo optimization techniques. Developed in order to find global optima in very complex spaces [16], these techniques introduce randomness as a means to avoid local minima traps. Many variants have been developed (e.g., random walk and simulated annealing [17]). The basic exploration process typically relies on successive transition tests using the Metropolis criterion (see Section IV-B). Note also that the probabilistic conformational roadmap [18] developed to explore molecularenergy landscapes in computational biology applications integrates a similar transition test in the Probabilistic RoadMap (PRM) framework [19].

\section{MINIMAL WORK PATHS}

This section introduces the mechanical work criterion to measure path quality in a space that is mapped by a given cost function. Paths that are optimal according to this criterion are called Minimal Work (MW) paths. The T-RRT algorithm presented in the next section tends to produce such MW paths, as shown by the theoretical analysis and the experimental results in Sections V and VI, respectively. First, we introduce the notion of MW paths and illustrate how this criterion generally yields more natural solution paths (i.e., paths following well the low-cost valleys of the costmap) compared with other existing path-quality measures.

\section{A. Notation}

Let us consider a system with a configuration space $\mathcal{C}$, possibly constrained by "binary" obstacle regions. Let us also consider a cost function $c: \mathcal{C} \rightarrow \mathbb{R}_{+}^{*}$ mapping this space, i.e., a $\operatorname{cost} c(q)>0$ can be computed for each $q \in \mathcal{C}$. This cost function $c$ is assumed to be continuous. A path $\mathcal{P}$ of length $l$ is represented by a unit-speed parametric function ${ }^{2} \tau:[0, l] \rightarrow \bigodot$

\footnotetext{
${ }^{2}$ This representation assumes that the parameterized curve that represents the path is regular, which simplifies the notation.
} 
with $\tau(s)=q_{s} \in \mathcal{P}$. Then, we define the parametric cost function $v:[0, l] \rightarrow \mathbb{R}_{+}^{*}$ of a path as $v(s)=c \circ \tau(s)=c\left(q_{s}\right)$.

\section{B. Classical Path-Quality Measures}

Several criteria have been proposed to evaluate the quality of a path from its parametric cost function, e.g., maximal cost [5], average cost [5]-[7], or costs sum over discrete path configurations [5], [8] (as a way to approximate the integral of the cost along the path). The maximal cost criterion is the most limited one, since it only relies on a point value of the parameterized cost function. The average cost can also be misleading, since it does not account for path length (a path that involves many detours inside a low-cost region will have an average cost smaller than a path that goes straight through this region). Thus, the integral of the cost along a path appears to be a more reliable criterion. It is mathematically defined as follows:

$$
S(\mathcal{P})=\int_{0}^{l} v(s) d s .
$$

A discrete approximation of the integral leads to

$$
S(\mathcal{P}) \sim \frac{l}{n} \sum_{k=0}^{n-1} v\left(s_{k}\right), \text { with } s_{k}=\left(\frac{k}{n-1}\right) l .
$$

In what follows, optimal paths according to the Integral of the Cost criterion are called IC paths. The next section introduces an alternative way to measure path quality based on the notion of mechanical work. This alternative technique will then be compared with IC criterion in Section III-D.

\section{Mechanical Work of a Path}

The key idea is that positive variations of the parametric cost function can be seen as forces acting against motion, and thus, producing mechanical work. We propose to use this loss of "energy" induced by the mechanical work to measure the quality of a path. In the case of negative variation of costs, the system loses no energy. Then, a small penalty proportional to the distance is added in order to favor shortest paths of equal mechanical energy. Based on this principle, the mechanical work of a path is defined as follows:

$$
W(\mathcal{P})=\int_{\mathcal{P}^{+}} \frac{\partial v}{\partial s} d s+\epsilon \int_{\mathcal{P}} d s
$$

where $\mathcal{P}^{+}$represents the portions of path with positive slopes (i.e., where the parametric cost function is strictly increasing), and $\epsilon$ is assumed to be very low compared with cost values.

The continuous expression of $W$ in (1) can be transformed into a discrete formulation expressed from the local extrema values along the path:

$$
\begin{aligned}
W(\mathcal{P}) & =\sum_{i}\left(v\left(\beta_{i}\right)-v\left(\alpha_{i}\right)\right)+\epsilon l \\
& =\sum_{i} \Delta v_{i}^{+}+\epsilon l
\end{aligned}
$$

where $\alpha_{i}$ and $\beta_{i}$ are consecutive minima and maxima of the costs along the paths, and $\Delta v_{i}^{+}=v\left(\beta_{i}\right)-v\left(\alpha_{i}\right)$ are the positive



Fig. 2. Decomposition of a path into portions of monotonic cost variation. $\alpha_{i}$ and $\beta_{i}$ correspond to local minima and maxima, respectively. (Right) Mechanical work is the sum of positive cost variations between consecutive extrema plus a small value $\epsilon l$ proportional to the path length.



Fig. 3. MW solution paths. (a) Paths are computed by using the $A^{*}$ algorithm within a 2-D grid discretizing the space. The examples illustrate (b) down-todown, (c) top-to-top, and (d) top-to-down queries, respectively.

variations between two consecutive extrema (see Fig. 2). The mechanical work of a path is simply obtained by summing up the positive differences between extrema of its parameterized cost function and adding $\epsilon l$ in order to favor shortest paths among the ones having equally positive cost variations. Paths that minimize the mechanical work for a given query are called MW paths.

Fig. 3 shows examples of MW paths for several queries on a 2-D hilly costmap. The paths were computed by using a standard $A^{*}$ search performed on a grid discretizing the 2-D landscape. As one can see, the shapes of the MW paths appear to be suitable in the sense that they follow as much as possible the low-cost regions of the space. In order to better state the pertinence of the mechanical work criterion, we first compare it with the IC criterion discussed in the next section. Then, we state some interesting properties of the MW criterion in Section III-E.

\section{Minimal Work versus Integral of the Cost}

This section compares the optimal solutions for the integral of the cost $S$ (IC paths) and for the mechanical work $W$ (MW paths) on representative cost spaces. 




(a)

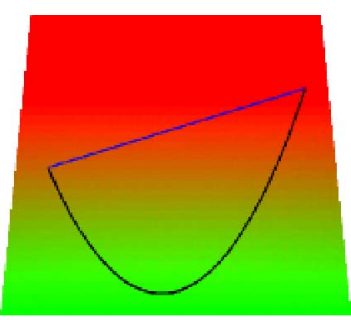

(b)
Fig. 4. Straight-line MW path (blue) and two different IC paths for two different inclinations of the plane that represent the cost function.

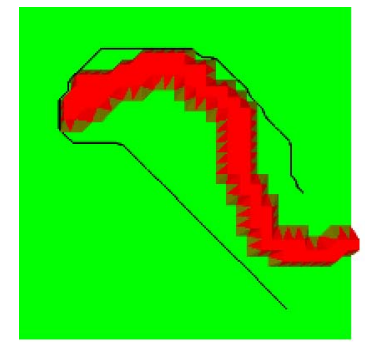

(a)

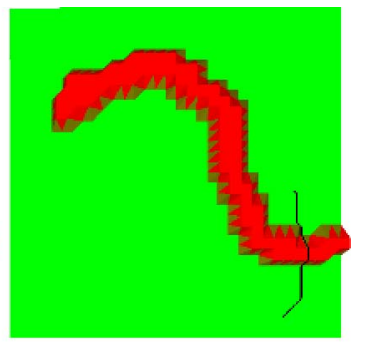

(b)
Fig. 5. High-cost barrier problem. (a) MW path. (b) IC path.

1) Constant Slope: Let us first consider the example of a planar landscape with a constant slope. In this simple case, IC solutions can be numerically characterized from calculus of variations. As shown in Fig. 4, the solutions obtained for two different slopes show that IC paths (in black) are not intuitive and, moreover, depend on the plane inclination. In contrast, in both cases, the MW path is the trivial straight-line path (in blue). Indeed, the cost of MW paths is always lower bounded by the cost variation between the initial and final configurations. In situations for which the query configurations can be connected through a set of paths having a monotonic cost variation (as for the specific case of a constant slope landscape), the MW path will be the shortest one among the set of minimal cost variation paths. This yields a straight-line solution for the planar slope example.

2) High-Cost Barrier: This example corresponds to a flat cost surface with a high-cost barrier that should be preferably avoided (see Fig. 5). In this case, the MW path is the shortest path to get around the barrier [see Fig. 5(a)], while the IC solution is a direct path that crosses the barrier [see Fig. 5(b)]. This example highlights another possibly negative feature of the integral of the cost criterion that may favor undesirable paths with short high-cost portions.

3) Hilly Costmap: In this more complex example, solution paths have to go through a saddle point to link the query configurations located at two opposite corners of the hilly landscape (see Fig. 6). The MW path makes necessary detours to follow low-cost valleys of the space. In contrast, the IC solution prefers shortest paths at the expense of local high costs [circled in blue in Fig. 6(b)]. As can be seen in the parameterized cost functions of the two kinds of optimal paths (see Fig. 7), the cost profile of the IC path (red) is globally much higher than the one of the



(a)



(b)
Fig. 6. Hilly costmap problem. (a) MW path. (b) IC path.

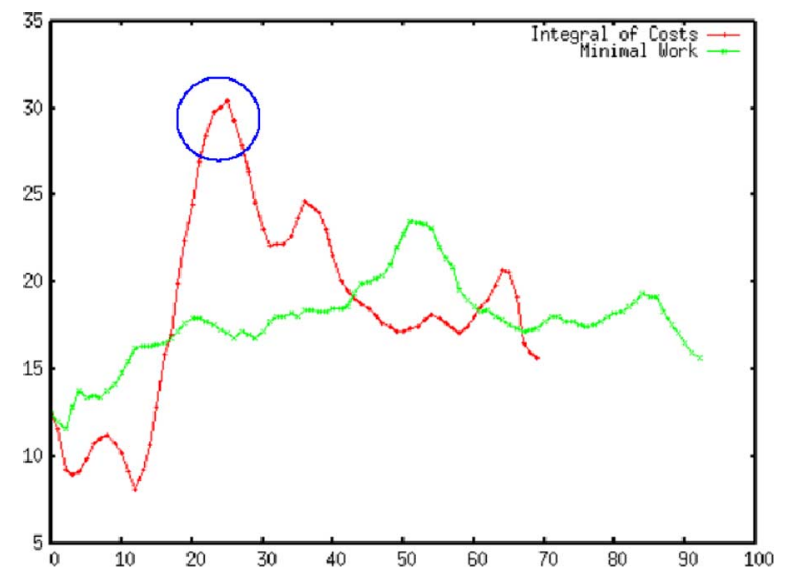

Fig. 7. Parameterized cost functions of the MW path (green) and the IC path (red) shown in Fig. 6(a) and (b).

TABLE I

MW AND IC OPTIMAL PATHS OF FIG. 6 COMPARED WITH A REFERENCE StRaight-Line SOLUTION

\begin{tabular}{l|ccccc}
\hline & Length & $C_{\text {ave }}$ & $C_{\max }$ & $S$ & $W$ \\
\hline MW path & 186 & $\mathbf{1 7 . 9}$ & $\mathbf{2 3 . 6}$ & 3324 & $\mathbf{1 5 . 9}$ \\
IC path & 139 & 18.6 & 30.4 & 2592 & 32.5 \\
Str. Line & 127 & 22.6 & 39.6 & 2877 & 41.0 \\
\hline
\end{tabular}

MW path (green). This observation is particularly true when the IC path goes through the high-cost region avoided by the MW path.

Finally, Table I compares the costs of the two solutions with respect to various path-quality measures. It shows that both the average and maximum costs are better for the MW path than for the IC path. Indeed, the IC path characteristics are intermediate between the ones of the MW path and of a simple straight-line path, not biased to avoid high-cost regions.

These results highlight some interesting features of the MW criterion. Compared with IC paths, MW paths avoid steep variations of the cost function. This may be particularly important in applications, such as outdoor navigation (to avoid high-slope motions), or computational biology (to minimize the crossing of high-energy barriers). Besides, in the presented cases, MW paths look more natural. In the next section, we present some additional properties of MW paths for a deep understanding of the mechanical work criterion. 


\section{E. Minimal Work Path Properties}

1) Negative Slopes Minimization: Section III-C states that to minimize the mechanical work means to minimize the amount of positive cost variations. A first property is that, between two given configurations, it also leads to minimize the negative cost variations. Indeed, the total amount of cost variations along the path can be expressed as follows:

$$
v(l)-v(0)=\sum_{i} \Delta v_{i}^{+}+\sum_{j} \Delta v_{j}^{-}
$$

where $\Delta v_{i}^{+}$and $\Delta v_{j}^{-}$are the intervals of positive and negative cost variation, respectively. Using (2), we obtain

$$
W(\mathcal{P})=v(l)-v(0)+\epsilon l+\sum_{j}\left|\Delta v_{j}^{-}\right| .
$$

Because $v(l)$ and $v(0)$ are constants and $\epsilon l$ is small relative to cost values, (3) states that to minimize $W$ is equivalent to minimize the last term in the right-hand side of the equation, that is, the total amount of negative cost variations.

2) Cost Variations Minimization: Since the MW path $\mathcal{P}$ minimizes both positive and negative cost variations, $\mathcal{P}$ is indeed the path that minimizes any cost variation between two given configurations. Let $V(\mathcal{P})$ be a function that sums positive and negative variations

$$
V(\mathcal{P})=\sum_{i} \Delta v_{i}^{+}+\sum_{j}\left|\Delta v_{j}^{-}\right| .
$$

Using (2) and (3), we get

$$
V(\mathcal{P})=2 W(\mathcal{P})-(v(l)-v(0)+2 \epsilon l) .
$$

Thus, the ordering of the paths remains the same regardless of the criterion ( $V$ or $W$ ), which, indeed, means that they are equivalent. However, we will keep the formulation of MW path, since this notion facilitates the analysis of the T-RRT algorithm.

3) Reversibility of Minimal Work Paths: Let ${ }^{-1} \mathcal{P}$ be the reverse path of $\mathcal{P}$. Since the parametric cost functions $v$ and ${ }^{-1} v$ have opposed variations, i.e., $\Delta^{-1} v^{+}=\left|\Delta v^{-}\right|$, we have

$$
W\left({ }^{-1} \mathcal{P}\right)=\sum_{j}\left|\Delta v_{j}^{-}\right|+\epsilon l
$$

and using (3), we get

$$
W\left({ }^{-1} \mathcal{P}\right)=W(\mathcal{P})+v(0)-v(l) .
$$

Consequently, the mechanical work of a path is equal to the mechanical work of its inverse, except for a constant. This property allows us to speak about the MW path between two configurations with no need to orient the path.

\section{TRANSITION-BASED RAPIDLY EXPLORING RANDOM TREE}

\section{A. Main Algorithm}

The T-RRT algorithm combines the advantages of two methods. First, it benefits from the exploratory strength of RRT-like algorithms, which result from their expansion bias toward large Voronoi regions of the space. Additionally, it integrates features of stochastic optimization methods developed to compute global

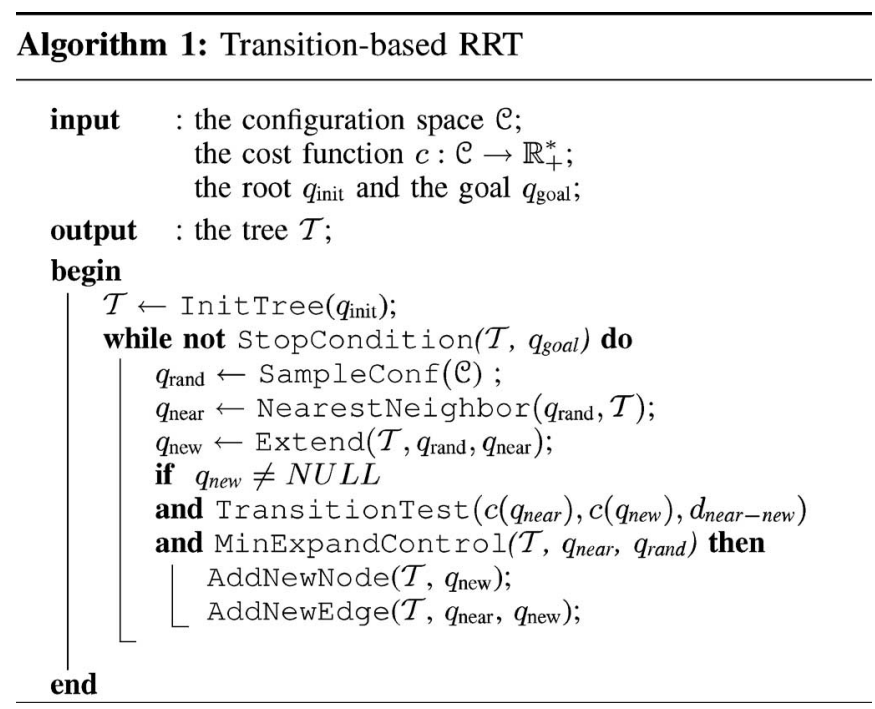

minima in complex spaces: It uses transition tests to accept or reject potential states.

Algorithm 1 shows the pseudocode of the T-RRT planner. Similar to the Extend version of the basic RRT algorithm [20], a randomly sampled configuration $q_{\text {rand }}$ is used to determine both the nearest tree node $q_{\text {near }}$ to be extended and the extension direction. The extension from $q_{\text {near }}$ is performed toward $q_{\text {rand }}$ with an increment step $\delta$. In the case of T-RRT, $\delta$ has to be small enough to avoid cost picks to be missed by the linear interpolation between $q_{\text {near }}$ and $q_{\text {new }}$. This stage also integrates collision detections in the presence of "binary obstacles." Thus, if the new portion of path leads to a collision, a null configuration is returned and the extension fails, independently of the associated costs. This extension process ensures the bias toward unexplored free regions of the space. The goal of the second stage is to filter irrelevant configurations regarding the search of low-cost paths before inserting $q_{\text {new }}$ in the tree. Such filtering is performed by the TransitionTest function. It relies on the Metropolis criterion commonly used in stochastic-optimization methods. This test integrates a selftuning technique in order to automatically control its filtering strength and, thus, to ensure continuous growth of the tree. Finally, the MinExpandControl function forces the planner to maintain a minimal rate of expansion toward unexplored regions of the space and avoids possible blocking situations during the search. The following sections detail the TransitionTest and MinExpandControl functions.

\section{B. Transition Test}

The TransitionTest function is presented in Algorithm 2. First, configurations with a higher cost than the maximum cost threshold $c_{\max }$ are filtered. The probability of acceptance of a new configuration is defined by comparing its cost $c_{j}$ relatively to the cost $c_{i}$ of its parent in the tree. This test is based on the Metropolis criterion initially introduced in statistical physics and molecular modeling. The transition probability 


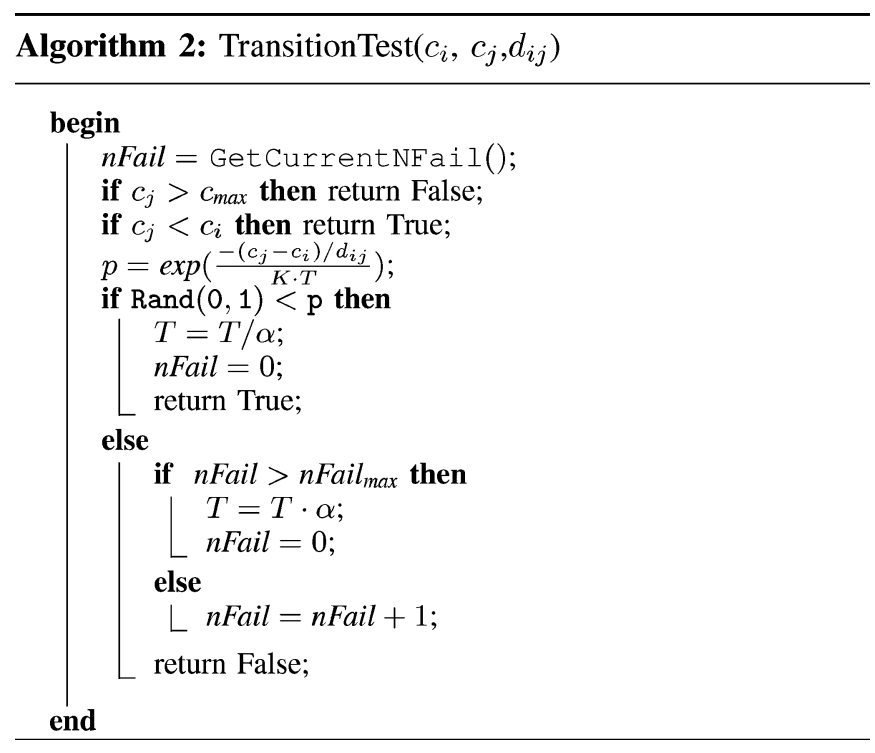

$p_{i j}$ is defined as follows:

$$
p_{i j}= \begin{cases}\exp \left(-\frac{\Delta c_{i j}}{K T}\right), & \text { if } \Delta c_{i j}>0 \\ 1, & \text { otherwise }\end{cases}
$$

where we have the following:

1) $\Delta c_{i j}=\left(c_{j}-c_{i}\right) / d_{i j}$ is the slope of the cost, i.e., the cost variation divided by the distances between the configurations. $^{3}$

2) $K$ is a constant value used to normalize the expression. It is based on the order of magnitude of the considered costs. $K$ is taken as the average cost of the query configurations, since they are the only cost values known at the beginning of the search process.

3) $T$ is a parameter called temperature that is used to control the difficulty level of transition tests, as further explained in the following. Note that the term temperature is employed in analogy with methods in statistical physics, but in our case, it does not have any physical meaning.

Using this transition probability, downhill transitions are automatically accepted, whereas for uphill transitions, the chance of acceptance decreases exponentially with the cost increment.

1) Temperature Parameter: $T$ is a key parameter of the algorithm, since it defines the level of difficulty of a transition for a given cost increment. Low temperatures limit the expansion to slightly positive slopes. In contrast, higher temperatures enable to climb the steeper slopes. Within methods that involve the Metropolis criterion, the temperature is usually kept constant (e.g., Monte Carlo search) or decreases gradually as the search progresses (e.g., simulated annealing). In our algorithm, this parameter is dynamically tuned according to the information acquired during the exploration.

2) Adaptive Tuning: The TransitionTest function performs an adaptive tuning of the temperature during the search

\footnotetext{
${ }^{3}$ Contrarily to classical Monte Carlo methods, the cost variation is normalized by the distance to the previous state, since this distance is not necessarily constant.
}

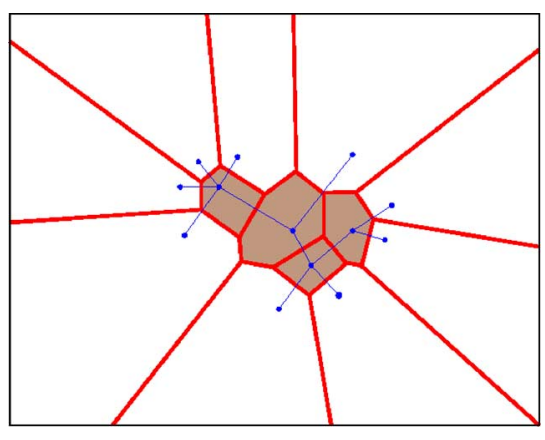

Fig. 8. Frontier nodes (in white regions) have a Voronoi region bounded by the space limits. On the contrary, the Voronoi region of nonfrontier nodes is bounded by the Voronoi region of other nodes (in brown/gray regions).

process (second stage of Algorithm 2). At the initialization, $T$ is set to a very low value (e.g., $\left.10^{-6}\right)$ in order to only authorize very easy positive slopes (and negative ones). Then, during the exploration, the number $n F$ ail of consecutive times the Metropolis criterion discards a configuration is recorded and used for temperature tuning. When the T-RRT search reaches a maximal number of rejections $n F a i l_{\max }$, the temperature is multiplied by a given factor $\alpha$. Each time an uphill transition test succeeds, the temperature is divided by the same factor $\alpha$. Thus, the temperature automatically adapts itself, such that an extension that corresponds to a positive cost variation is performed in average every $n F a i l_{\max }$ times. The influence of parameters $\alpha$ and $n F a i l_{\text {max }}$ is analyzed in Section VI.

\section{Minimal Expansion Control}

The adaptive temperature tuning introduced earlier ensures a given success rate of positive slope transitions. A possible side effect may appear when the tree expansion toward unexplored regions remains slow, and the new nodes contribute only to refine already explored regions. We discuss in the following this issue and explain how the MinExpandControl function overcomes this problem.

1) Exploration Versus Refinement: The behavior of the RRT expansion can be explained by distinguishing two types of nodes [21]. Frontier nodes are the external nodes of the tree with a Voronoi region bounded by the space limits, whereas nonfrontier nodes are the internal ones, whose Voronoi region is entirely bounded by the Voronoi region of the other nodes (see Fig. 8). Thus, the extension of a frontier node tends to explore new regions of the space, and the extension of a nonfrontier node only refines the existing tree. The problem of unbalanced refinement and exploration modes was addressed in [21] and [22] for standard RRTs. However, for T-RRT, the interaction between these two kinds of extensions is more subtle than for the basic RRT. Indeed, situations occur where the temperature is stabilized by new nonfrontier nodes, which refine the tree in easier regions of the space; however, the expansion toward new regions requires the development of frontier nodes. Fig. 9(a) illustrates this issue with an example of a tree, whose expansion has been slowed down by the too-frequent insertion of nonfrontier nodes. 


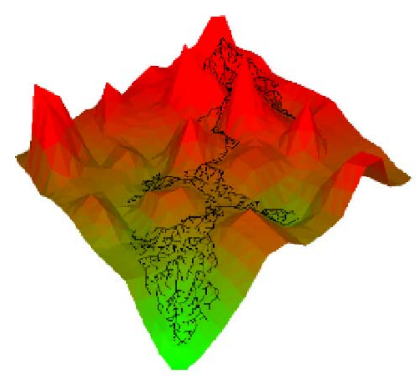

(a)

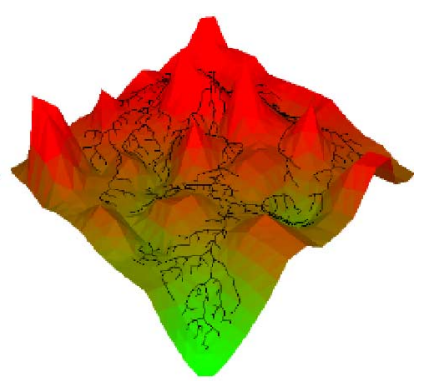

(b)
Fig. 9. Impact of the minimal expansion control on the T-RRT algorithm. (a) Without control, the insertion of nonfrontier nodes tends to slow down the exploration by decreasing the temperature. (b) With control, the planner is forced to keep to explore new regions of the space.

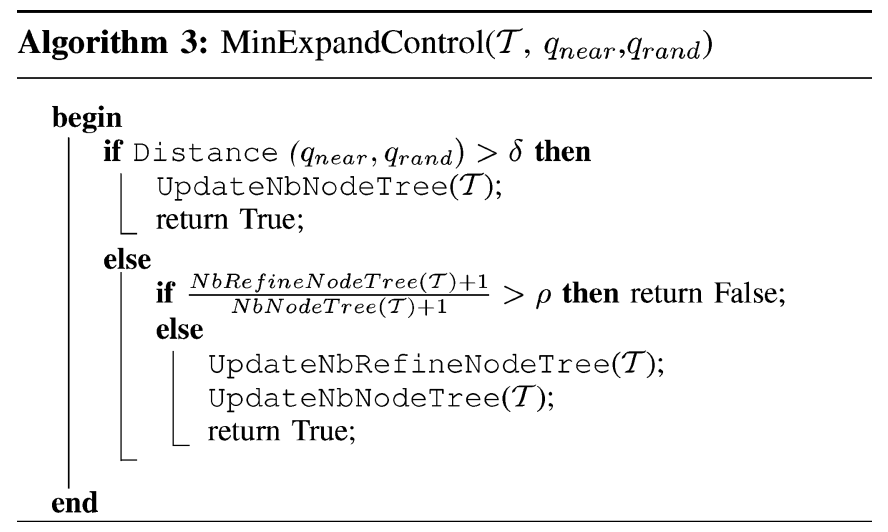

Fig. 9(b) shows the tree obtained by using the minimal expansion control detailed in the following.

2) Minimal Exploration Rate: The proposed solution is to force the planner to explore new regions by controlling the ratio between exploration and refinement steps. Note that as long as the tree coverage remains limited compared with the size of the space, nonfrontier nodes have a Voronoi region that is much smaller than the one of frontier nodes. Hence, extension steps can be estimated as refinements or expansions, which depend on the distance between $q_{\text {near }}$ and $q_{\text {rand }}$. For a large distance value, $q_{\text {near }}$ has greater chances to be a frontier node, whereas a small distance value corresponds most probably to the case of a nonfrontier node extension. The control of minimal exploration rate is performed by the MinExpandControl function presented in Algorithm 3. If the distance $q_{\text {near }}-q_{\text {rand }}$ is greater than the expansion step $\delta, q_{\text {new }}$ is considered to participate in the tree expansion, and it is inserted in the data structure. Otherwise, $q_{\text {new }}$ is considered to participate in the tree refinement. The configuration is not inserted in the tree if it makes the ratio of nonfrontier nodes be greater than a given maximal value $\rho$. The influence of this parameter is further discussed in Section VI.

\section{THEORETICAL ANALYSIS OF T-RRT}

\section{A. T-RRT and Minimal Work Path}

This section analyzes the relationship between T-RRT and the notion of MW path introduced in Section III. An important property is obtained first for the simplified case of a discrete

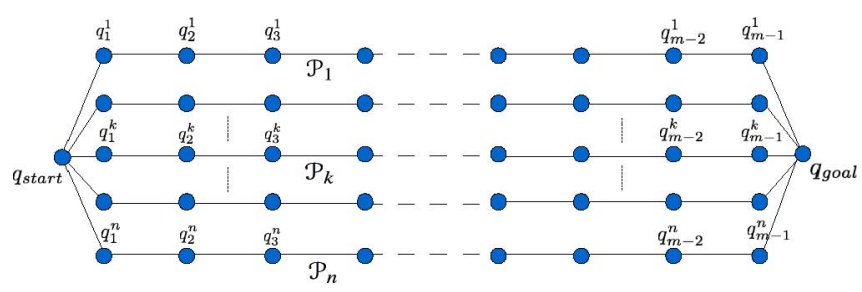

Fig. 10. Case of $n$ equal-length paths. With T-RRT, the branches with the lowest mechanical work have the highest chances to reach the goal first.

search process. Then, we discuss the extension of this result to the general case of the T-RRT search.

1) Simplified Case: Let us consider a path search within a discrete set of $n$ equal length possible paths, each one defined by a sequence of $m$ edges and $m+1$ nodes (see Fig. 10). Using a T-RRT scheme, each expansion of a given path requires the path to be selected and the associated transition test to succeed. Thus, the probability $P_{k}$ of a given path $\mathcal{P}_{k}$ to be entirely developed in $m$ iterations is equal to

$$
P_{k}=\prod_{i \in[1, m]}{ }^{e} p_{i}^{k}=\prod_{i \in[1, m]}\left({ }^{s} p_{i}^{k}\right)\left({ }^{t} p_{i}^{k}\right)
$$

where ${ }^{e} p$ denotes the probability for a given node to be extended, ${ }^{s} p$ is the probability to be selected, and ${ }^{t} p$ is the probability to have an accepted transition. In addition, we assume that the paths have equal chances of being extended at each step (i.e., the node-selection process is not biased by Voronoi regions), i.e.,

$$
P_{k}=\frac{1}{n^{m}} \prod_{i \in[1, m]}{ }^{t} p_{i}^{k} .
$$

If the transition probability depends only on the transition tests (i.e., the MinExpandControl is omitted), we get

$$
P_{k}=\frac{1}{n^{m}} \prod_{j} e^{\left(-\Delta v_{j}^{k+}\right) / K T_{j}} .
$$

Moreover, if we assume that the temperature remains constant during the expansion, we have

$$
P_{k}=\frac{1}{n^{m}} e^{1 / K T} e^{-\sum_{j} \Delta v_{j}^{k+}}
$$

where $\Delta v_{j}^{k+}$ are summed over the positive variations of cost along the path $k$. Finally, since $\epsilon l$ is negligible in the mechanical work expression, we get

$$
P_{k}=\frac{1}{n^{m}} e^{1 / K T} e^{-W\left(\mathcal{P}_{k}\right)} .
$$

Since $\left(1 / n^{m}\right) e^{1 / K T}$ is the same for all the paths, we obtain an important property for this simplified version: The paths with the lowest mechanical work have the highest probability of reaching the goal first.

2) General Case: One first assumption made in the analysis earlier is that each branch has an equal chance of being chosen for the expansion. In practice, the various paths developed by the T-RRT algorithm (from the root node to each leaf) are not spatially independent. Each branch expansion tends to increase 
its global Voronoi region and, thus, increases the chance for its nodes to be selected at the next iteration. This process reinforces the extension of the paths with the most favorable mechanical work and increases the convergence of the planner toward lower cost solutions.

The simplified version also assumed that the temperature is constant. This parameter affects each path in the same way. Thus, we can argue that the property remains valid, even when $T$ varies during the search.

Finally, whereas the aforementioned property is established for the discrete case of equal-length solution paths, T-RRT search is performed among an infinite number of variable-length paths. Since shortest paths require less expansion steps to connect the queries, it is not possible to guarantee that paths of lower cost have always better chances to reach the goal first. However, as one can see from (7), the mechanical work of a path affects exponentially its chances of success. This reveals how strongly the T-RRT exploration is implicitly biased toward solution paths of low mechanical work.

\section{B. Probabilistic Completeness}

The T-RRT algorithm is a probabilistically complete planner [19]. This property is directly inherited from the probabilistic completeness of the RRT planner (see [9, Sec. IV]). The only difference is that in the present case, the extension steps can be rejected because of the transition tests, even in the case of a convex, open, $n$-dimensional subset of an $n$-dimensional configuration space. However, we argue that the success probability of the transitions is always strictly positive, since the cost function takes finite values in this subset, and thus, the cost variations are bounded. As a result, the planner converges eventually toward an entire coverage of the considered subset, and the transition tests affect only the convergence rate of the algorithm.

\section{EXPERIMENTAL RESULTS}

A large set of experiments has been conducted to evaluate the performance of the planner. First, the general behavior of the method is presented on various problems. Second, its performance is compared with that of the existing methods to highlight the good quality of the T-RRT solutions. Finally, we investigate the influence of some intrinsic parameters on the overall efficacy of the method. All the algorithms have been implemented within the path-planning software Move3D [23]. The performance results summarized in the tables are values averaged over ten runs.

\section{A. General Performance}

A variety of problems are proposed to illustrate the generality of the method. The examples vary not only in the geometrical complexity and the configuration space dimensionality but in the nature of the cost function as well. Two settings of T-RRT are considered: A greedy version of the planner referred to as T$\mathrm{RRT}_{g}$ that takes $n$ Fail $l_{\text {max }}=10$, and a tempered version, which is referred to as T-RRT ${ }_{t}$, with $n F a i l_{\text {max }}=100$. The latter leads to higher quality solution paths but is more computationally expensive. We used $\alpha=2$ in all the examples. The results obtained


Fig. 11. (a) and (b) Construction process of the transition-based RRT planner. The solution path (c) is close to the optimal one (d) computed from a space discretization.

TABLE II

COMPARATIVE RESUltS FOR THE COSTMAP PROBLEM

\begin{tabular}{|c|c|c|c|c|c|c|}
\hline & Length & $C_{\text {ave }}$ & $C_{\max }$ & $S \times 10^{-3}$ & $W$ & Time \\
\hline RRT & 148 & 22.6 & 41.2 & 3.3 & $45.1(36.9)$ & 0.1 \\
\hline $\mathrm{T}^{-R_{R T}}{ }_{g}$ & 182 & 18.2 & 25 & 3.9 & $28.0(19.7)$ & 0.9 \\
\hline $\mathrm{T}^{-R R T_{t}}$ & 214 & 17.1 & 23.2 & 4.0 & $23.1(16.9)$ & 11.0 \\
\hline MW path & 186 & 17.9 & 23.6 & 3.3 & 15.9 & - \\
\hline IC path & 139 & 18.6 & 39.6 & 2.6 & 41.0 & - \\
\hline $\bar{T}$ Thresh. & $\overline{157-192}$ & $\overline{17.8-20.9}$ & $\overline{23} .7-32.7$ & $\overline{3.3-3.4}$ & $27-30-(21-24)$ & $0 . \overline{3}-329$ \\
\hline hRRT & 155 & 20.9 & 33.3 & 3.2 & $41.4(32.9)$ & 0.6 \\
\hline
\end{tabular}

with the basic RRT planner are given as references. The tables also present comparative results with two existing cost-based methods that will be discussed latter.

The first set of experiments is performed on the 2-D cost space shown in Fig. 1. In this example, the solution paths have to go through a saddle point to link the query configurations located at two opposite corners of the landscape. Fig. 11 shows snapshots of the exploration tree and the solution path found [see Fig. 11(c)], which is close to the optimal one [see Fig. 11(d)]. Table II presents the characteristics of the paths obtained with each planner. ${ }^{4}$ It also provides values for the MW and IC optimal paths (computed with an $A^{*}$ search within a $128 \times 128$ grid discretizing the landscape).

The mechanical work of solutions obtained by the different methods is reported in the $W$ column. The numbers in parentheses integrate the effect of some local smoothing of the solution path with a simple procedure based on the shortcut algorithm [14]. As one can see from Table II, the mechanical work of the reference RRT path is almost three times higher than for the optimal MW solution, and smoothing does not successfully get close to the optimal value (36.9 versus 15.9 ). In comparison, the mechanical work of the path obtained with the tempered version of T-RRT is only $45 \%$ higher than for the MW path, and it

\footnotetext{
${ }^{4}$ In the case of RRT, since there is no obstacle in the scene, connection attempts to the goal are only performed when $d\left(q_{\text {new }}, q_{\text {goal }}\right)<15 \delta$ to avoid getting a trivial straight-line solution.
} 


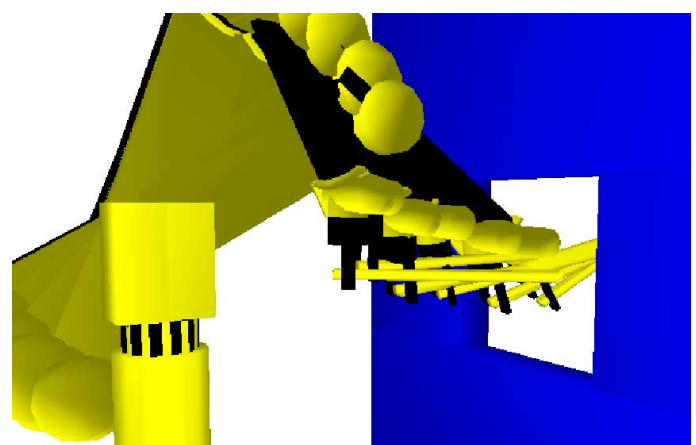

Fig. 12. Stick-extraction problem. A 6-DOF manipulator arm has to extract a stick from a hole. The T-RRT solution path keeps the stick horizontal to maximize its distance to the obstacles.

TABLE III

COMPARATIVE RESULTS FOR THE STICK-EXTRACTION PROBLEM

\begin{tabular}{|c|c|c|c|c|c|c|}
\hline & Length & $C_{\text {ave }}$ & $C_{\max }$ & $S$ & $W$ & Time \\
\hline RRT & 55.3 & 6.6 & 377 & 363 & 1196 & 1.5 \\
\hline $\mathrm{T}^{-\mathrm{RRT}_{g}}$ & 53.9 & 0.4 & 1.0 & 21.8 & 1.9 & 7.4 \\
\hline $\mathrm{T}-\mathrm{RRT}_{t}$ & 51.5 & 0.3 & 0.9 & 17.4 & 1.1 & 32.8 \\
\hline Thresh. & $53.2-54 . \overline{9}$ & $\overline{0} . \overline{6-3} \overline{2}$ & $\overline{1} . \overline{6}-40.1$ & $3 \overline{2.0}-\overline{1} 7 \overline{2}$ & $\overline{4} .2-19 \overline{6}$ & $\overline{1} . \overline{6-2 . \overline{7}}$ \\
\hline hRRT & 53.5 & 5. & 341 & 265 & 786 & 3.0 \\
\hline
\end{tabular}

becomes only $6 \%$ higher than the optimal value after smoothing. Most important, the overall shape of the T-RRT solution is very close to the optimal-MW path and follows the same low-cost regions. In addition, note that the relatively slight loss of path quality of the greedy version is compensated by a much smaller computing time $(0.9 \mathrm{~s}$ versus $11.0 \mathrm{~s})$. Comparative results obtained with other existing costmap planners (Thresh. and hRRT rows in Table II) are discussed in Section VI-B.

In the next experiment, a six-degrees-of-freedom (DOF) manipulator arm is carrying a stick in a 3-D workspace with obstacles (see Fig. 12). Here, the goal is to extract the stick from a hole, while keeping the stick as far as possible from the obstacles. Thus, the cost function considered here is the inverse of the distance between the stick and the obstacles. Results are presented in Table III.

The costs of the T-RRT solution paths are considerably lower than the ones of RRT. This shows the effectiveness of the planner to find low-MW paths in higher dimensional spaces. T-RRT solutions tend to keep the stick horizontal during its extraction from the hole in order to remain as far as possible from the obstacles, whereas the basic RRT planner produces erratic paths. Once again, the slight loss of path quality of the greedy version of the T-RRT (1.9 versus 1.1) is compensated by a significant speed up (7.4 s versus $32.8 \mathrm{~s}$ ).

The third scenario involves the same manipulator arm that carries a sensor with a spherical extremity for the inspection of the surface of a car part. The goal here is to keep the sensor close to the surface of the car part during the motion, in order to satisfy the requirements for the surface following task (see Fig. 13 and Table IV). Note that for such a scenario, where the robot is subject to task-space constraints, specific path-planning schemes also exist (e.g., [24]).

As to be expected, the T-RRT computing time is higher than the one of RRT because to compute a collision-free path with

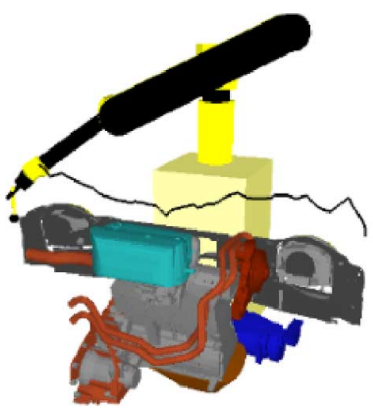

(a)

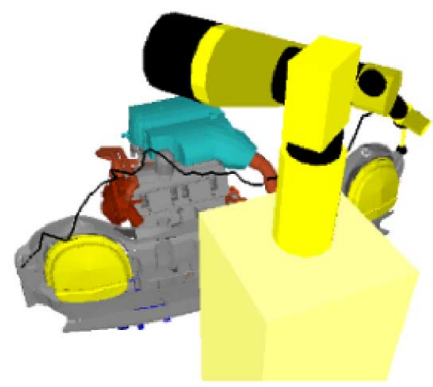

(b)
Fig. 13. Car-part-inspection problem. The path for a 6-DOF arm that manipulates a sensor (black sphere), which needs to remain close to the surface during the inspection task is shown.

TABLE IV

COMPARATIVE RESUlts FOR THE CAR-PART-INSPECTION PROBLEM

\begin{tabular}{|c|c|c|c|c|c|c|}
\hline & Length & $C_{\text {ave }}$ & $C_{\max }$ & $S \times 10^{-3}$ & $W$ & Time \\
\hline RRT & 3785 & 457 & 875 & 1730 & 1434 & 0.98 \\
\hline T-RRT $_{g}$ & 3515 & 24.4 & 87.5 & 85.8 & 400 & 16.4 \\
\hline $\mathrm{T}^{\mathrm{RRRT}} \mathrm{t}_{t}$ & 3396 & 4.3 & 28.1 & 14.6 & 187 & 206 \\
\hline $\bar{T}$ Tresh. & $-\overline{284} \overline{8-3 \overline{4}} \overline{2}$ & $-3 \overline{6}-1 \overline{8} 6-$ & $7 \overline{9}-4 \overline{3} 6$ & $\overline{1} 1 \overline{6}-5 \overline{2} 9^{-}$ & $\overline{447-6 \overline{35}}$ & $\overline{4} . \overline{3}-1 \overline{9}$ \\
\hline hRRT & 3127 & 253 & 452 & 792 & 943 & 18.0 \\
\hline
\end{tabular}

RRT and with no cost consideration is a much easier problem than to obtain a solution that minimizes the distance to the inspected surface. However, with regard to paths quality, the mechanical work of T-RRT $g$ and T-RRT $\mathrm{T}_{t}$ are 3.6 times and 7.7 times lower than the one of RRT, respectively. The average and maximal costs reported in Table IV are interesting indicators to get a better idea of the quality of the results, since they correspond directly to the average and maximal distances between the sensor and the part, respectively. For a distance reference, the diameter of the black sphere at the extremity of the sensor is $40 \mathrm{~mm}$. For T-RRT , the maximal cost corresponds approximately to twice this value, whereas the average distance is close to the sensor radius. In the case of T-RRT, solution paths follow the surface of the part so well that the maximal distance never exceeds the size of the sphere and the average one is about one tenth of this diameter.

Finally, the last scenario corresponds to a molecular model shown in Fig. 14. The task is to compute the pathway to extract the ligand (small molecule in red/dark) from the active site located inside a protein. This problem can be seen as a mechanical disassembly path-planning problem for the free-flying ligand [25]. Energetic constraints are translated into geometric ones by considering a steric model of the molecule, and a collision-detection algorithm [26] is applied as a geometric filter that rejects conformations with prohibitively high van der Waals (VdW) energy. The cost function considered for this problem is the inverse of the distance between the ligand and the protein. The interest of this molecular model is to provide a simple way to quantify the quality of the computed solution path. The ligand-free space can be simply dilated by shrinking the atoms radii. The results reported in Table $\mathrm{V}$ correspond to both RRT and T-RRT algorithms applied on the shrunk model shown in Fig. 14(b) (25\% of VdW radii). 


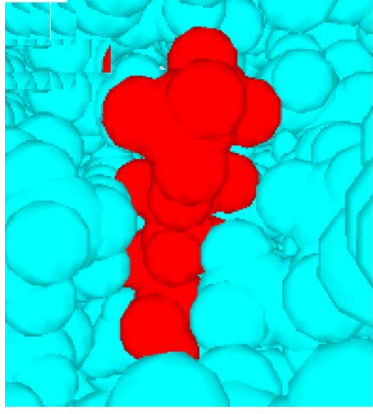

(a)

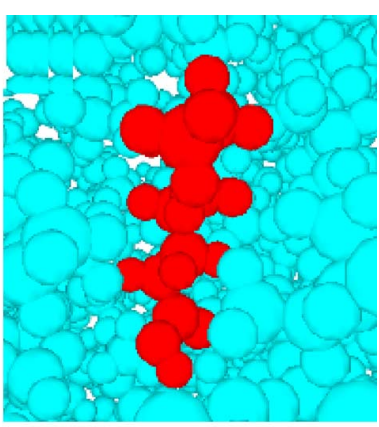

(b)
Fig. 14. Two representations of the same ligand-protein "disassembly" problem, with different VdW radii. (a) Maximal radius. (b) Shrunk radius. The goal is to compute paths that maximize the clearance and, thus, remain valid for large $\mathrm{VdW}$ radii.

TABLE V

COMPARATIVE RESUlTS FOR THE LIGAND-PROTEIN PROBLEM

\begin{tabular}{|c|c|c|c|c|c|c|c|}
\hline & Length & $C_{\text {ave }}$ & $C_{\max }$ & $S$ & $W$ & Time & $V d W_{m}$ \\
\hline RRT & 59 & 14 & 2236 & 826 & 471 & 0.7 & 25 \\
\hline T-RRT $_{g}$ & 62 & 0.4 & 1.0 & 22 & 1.25 & 8.39 & 65 \\
\hline$\underline{\mathrm{T}}-\mathrm{RRT}_{t}$ & 64 & 0.3 & 0.9 & 22 & 1.0 & 426 & 69 \\
\hline $\bar{T} \bar{r} \bar{r} \bar{h}$. & $-\overline{58}-\overline{6} 2$ & $. \overline{4}-\overline{7}$ & $\overline{1} . \overline{1-3} . \overline{7}$ & $\overline{25}-\overline{4} 2-$ & $1 . \overline{4}-\overline{4.3}$ & $\overline{4} . \overline{8}-7 \overline{3} .5^{-}$ & $\overline{3} 4-\overline{6} 2^{-}$ \\
\hline hRRT & 59 & 8.3 & 733 & 490 & 353 & 15.2 & 26 \\
\hline
\end{tabular}

The T-RRT solution paths have a much lower cost compared with the one computed by RRT. The higher clearance of the T-RRTs solutions are also quantified by the maximal VdW ratio indicated in the last row of the table. This maximal ratio was obtained by testing solution paths by increasing the VdW radii until a collision was detected between the ligand and the protein. While no growing is possible for the RRT solution, the T-RRT paths (computed with a $25 \%$ ratio) remain valid up to $65 \%$ and $69 \%$ growing, depending on the variant. These values are close to the maximal radius that allows the ligand to exit $(80 \%)$. The high clearance of T-RRT paths reflects their good quality with respect to the considered distance-based cost.

\section{B. T-RRT versus Existing Methods}

T-RRT has been compared with two existing cost-based planners: the maximal Threshold technique proposed by Ettlin and Bleuler [4] and the $h R R T$ of Urmson and Simmons [6]. Results obtained for the set of experiments with these planners are reported in the last two rows of Tables II-V. In the case of the threshold method, results are highly sensitive to the thresholdgrowing speed, and thus, reported data correspond to the extremal values obtained when varying this parameter in the range (1-100).

With regard to the mechanical work criterion, results show that $\mathrm{T}^{-\mathrm{RRT}_{t}}$ provides significantly better solutions than existing methods in all tests. Remarkably, T-RRT solutions are also better with respect to the IC criterion in the three more difficult problems, which involve a 6-D cost space.

The overall bad performance of the $h R R T$ method is due to the strong bias introduced by the heuristic that steers the exploration toward the goal, resulting in a poor exploratory ability, which makes it unable to circumvent high-cost regions and find higher quality paths. Comparatively, the threshold technique can pro-
TABLE VI

INFLUENCE OF THE $\alpha$ AND $n F a i l_{\text {max }}$ PARAMETERS

\begin{tabular}{|c|c|c|c|c|c|c|}
\hline \multicolumn{7}{|c|}{ Hilly costmap } \\
\hline & \multicolumn{3}{|c|}{ Time } & \multicolumn{3}{|c|}{$W$} \\
\hline & \multicolumn{3}{|c|}{$n$ Fail $_{\max }$} & \multicolumn{3}{|c|}{ nFail $_{\max }$} \\
\hline$\alpha$ & 10 & 100 & 1000 & 10 & 100 & 1000 \\
\hline 2 & 0.9 & 11.0 & 121 & 28.0 & 23.1 & 23.4 \\
\hline 10 & 0.7 & 7.1 & 93.4 & 32.1 & 26.6 & 24.4 \\
\hline 50 & 0.7 & 7.0 & 85.5 & 32.0 & 28.7 & 24.8 \\
\hline \multicolumn{7}{|c|}{ Stick extraction } \\
\hline \multicolumn{4}{|c|}{ Time } & \multicolumn{3}{|c|}{$W$} \\
\hline & \multicolumn{3}{|c|}{ nFail max $_{\max }$} & \multicolumn{3}{|c|}{ nFail $_{\max }$} \\
\hline$\alpha$ & 10 & 100 & 1000 & 10 & 100 & 1000 \\
\hline 2 & 7.4 & 32.8 & 226 & 1.9 & 1.1 & 1.1 \\
\hline 10 & 8.2 & 31.0 & 218 & 5.5 & 2.9 & 2.4 \\
\hline 50 & 7.0 & 29.8 & 226 & 3.5 & 3.3 & 1.2 \\
\hline \multicolumn{7}{|c|}{ Car part inspection } \\
\hline \multicolumn{4}{|c|}{ Time } & \multicolumn{3}{|c|}{$W$} \\
\hline & \multicolumn{3}{|c|}{ nFail $_{\max }$} & \multicolumn{3}{|c|}{ nFail $_{\max }$} \\
\hline$\alpha$ & 10 & 100 & 1000 & 10 & 100 & 1000 \\
\hline 2 & 16.4 & 206 & 2012 & 400 & 187 & 166 \\
\hline 10 & 14.3 & 171 & 1697 & 439 & 188 & 165 \\
\hline 50 & 15.9 & 167 & 1583 & 507 & 212 & 203 \\
\hline
\end{tabular}

TABLE VII

INFLUENCE OF THE $\rho$ PARAMETER

\begin{tabular}{l|cc|cc|cc}
\hline \multirow{2}{*}{$\rho$} & \multicolumn{2}{|c|}{ Hilly costmap } & \multicolumn{2}{c|}{ Stick extraction } & \multicolumn{2}{c}{ Car part inspection } \\
\cline { 2 - 7 } & Time & $W$ & Time & $W$ & Time & $W$ \\
\hline 1 & 420 & 19.6 & 30.3 & 1.1 & 201 & 192 \\
$1 / 2$ & 16.7 & 23.7 & 33.4 & 1.2 & 198 & 202 \\
$1 / 10$ & $\mathbf{1 1 . 0}$ & $\mathbf{2 3 . 1}$ & $\mathbf{3 2 . 8}$ & $\mathbf{1 . 1}$ & $\mathbf{2 0 6}$ & $\mathbf{1 8 7}$ \\
$1 / 100$ & 9.7 & 23.8 & 32.0 & 1.2 & 269 & 263 \\
\hline
\end{tabular}

vide solution paths whose quality is close to the one of T-RRT , , but its performance is highly sensitive to the parameter that regulates the variation speed of the threshold. Depending on the value of this parameter, the running time increases up to 1000 times for the costmap problem, the mechanical work increases up to 47 -fold for the stick-extraction problem, and both the running time and the mechanical work are notably affected by the threshold-speed value for the car-part-inspection problem. Furthermore, this sensitive parameter is problem-dependent and has to be tuned for each application, whereas T-RRT parameters remain robust to problem changes, as shown next.

\section{Influence of Intrinsic Parameters}

We now analyze the influence of the main parameters of the T-RRT algorithm. Experiments are performed on three problems that correspond to three different types of cost functions: the hilly costmap (see Fig. 11), the stick-extraction problem (see Fig. 12), and the car-part-inspection problem (see Fig. 13). The results are presented in Tables VI and VII. Bold values are the default settings used in previous tests.

1) Temperature Variation Control: $n$ Fail $l_{\max }$ and $\alpha$ are the two parameters that control the derivative of the temperature, and hence, the selectivity of the transition test (as explained in Section IV-B).

Table VI shows that $n F a i l_{\max }$ is an important parameter that determines the appropriate balance between time performance and solution-path quality. In the costmap problem, when $n$ Fail $_{\max }$ is increased by a factor of ten, the running time also increases 9-13-fold. Its influence on the runtime performance is less direct on the two manipulator problems (due to the 
additional cost of collision checking), even though the tendency is the same. Finally, note that higher values of $n F a i l_{\max }$ improve path quality but only up to a point: The quality increases when $n$ Fail $_{\max }$ varies from 10 to 100 but remains approximately constant from 100 to 1000 .

With regard to the $\alpha$ parameter, results show that it affects only slightly the behavior of the algorithm, even if higher values tend to increase the time performance, while decreasing the path quality. Overall, values $n F a i l_{\max }=100$ and $\alpha=2$ provide the best results for the three examples and are used as default setting for all tests.

2) Expansion Versus Refinement Control: Table VII presents result for various values of the $\rho$ parameter used in the MinExpandControl function to set the maximal ratio of refinement nodes.

In the first line of the table, $\rho=1$ means that the MinExpandControl function is inactive. The results for the 2-D hilly costmap highlight the importance of this function, the computing time being much higher when $\rho=1$. This example illustrates cases in which the refinement process slows down the exploration by decreasing the temperature. This effect is less visible in the two other examples, where refinement steps are less likely to happen because of the large size of the space. Results for the other settings (i.e., $\rho \neq 1$ ) are quite similar, which means that $\rho$ does not require to be tuned precisely. In all experiments, the default setting $\rho=1 / 10$ appears to be a good compromise between computing time and path quality.

\section{EXTENSIONS}

\section{A. Bidirectional T-RRT}

Similar to the bidirectional version of the RRT planner [9], a bidirectional T-RRT can be envisaged. However, a naive approach that would use the same transition test for both trees would lead to poor quality solutions. It would tend to create paths with consecutive downhill and uphill cost variations, which correspond to branches expanded from the init-tree and goal-tree, respectively, and may fail to find a more flat solution path of lower MW cost. A better alternative, using the property of Section III-E2, which states that the MW paths minimize any cost variations, is to modify transition tests in order to filter both positive and negative cost variations when expanding the two trees. This can be achieved easily by replacing the transition probability $p_{i j}$ of (6) by the expression $p_{i j}=\exp \left(-\left(\left|\Delta c_{i j}\right|\right) / K T\right)$. Preliminary results show that this approach performs well in problems where positive and negative cost variations for the best cost paths are globally of the same amplitude. However, in problems where the profile of the cost between query nodes is asymmetric, it turns out to reject too many configurations during the transition test, which degrades the performance. In this case, a method based on a more sophisticated transition test should be designed.

\section{B. Toward a Greedy Anytime T-RRT}

In this section, we discuss a possible extension of T-RRT for performance improvement in tricky situations, such as the one illustrated in Fig. 15. In this example, the large low-cost region has to be fully explored 1) for determining the need to cross

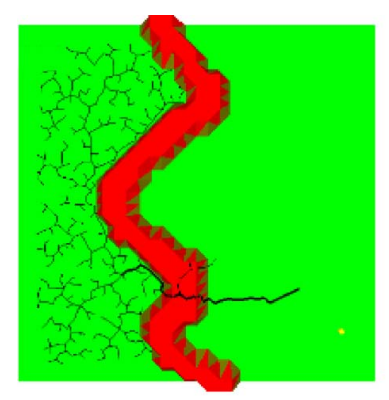

(a)



(b)
Fig. 15. Tricky problem for T-RRT. A large low-cost region has to be explored before deciding to cross the high-cost barrier. (a) Useless in or (b) leading to a better solution.



(a)

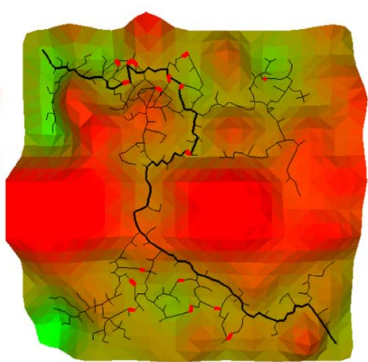

(b)
Fig. 16. (a) Initial tree built using a greedy T-RRT version. (b) Addition of cycles (in red) leads to higher quality paths.

the higher cost barrier or 2) to discover the low-cost passage that yields a better solution. In both cases, the greedy T-RRT $g$ version may rapidly cross the barrier and, thus, speed up the


may miss the preferred detour path in problem 2) for which a longer exploration is needed to find the passage. To keep the performance of an aggressive exploration, while avoiding this issue, we propose to combine the greedy version of the planner with a cycle-addition mechanism. The idea is to create cycles in the tree when good paths initially missed during the search are discovered afterward. The idea has been tested by using the technique described in [27] for cycle addition. Fig. 16 shows an initial tree built using a greedy version of T-RRT that goes through a medium cost region [circled in blue on Fig. 16(a)] that could have been avoided. The addition of cycles provides alternative paths and yields higher quality solutions [see Fig. 16(b)].

\section{CONCLUSION AND FUTURE WORK}

We have presented a sampling-based algorithm to compute paths in problems which involve high-dimensional cost spaces. The proposed method combines the exploratory strength of RRTs, with the efficiency of stochastic-optimization methods. It integrates an adaptive mechanism that helps to ensure a good performance for a large set of problems.

The notion of MW path has been proposed to quantify the quality of solution paths. By design, the proposed T-RRT algorithm computes paths that tend to satisfy such a quality property. 
A large set of experiments were performed to show the efficacy of the T-RRT planner.

Experimental results have shown that the planner is general enough to be applied, at least, to 6-D spaces constrained by obstacles. Future work concerns the application of T-RRT to new classes of problems, such as the integration of human-robot interaction constraints within path planning or the exploration of energy landscapes in computational biology problems. Extensions discussed in the previous section also need to be further explored for performance improvement. Furthermore, another direction is to incorporate in the planner other methods inspired by Monte Carlo optimization techniques, such as stochastic tunneling [28] or parallel tempering [29]. Finally, it would be interesting to test our approach on benchmark problems of the stochastic optimization community, since T-RRT could be used as a generic optimization tool and, in principle, applied to any metric cost space.

\section{REFERENCES}

[1] H. Choset, K. Lynch, S. Hutchinson, G. Kantor, W. Burgard, L. Kavraki, and S. Thrun, Principles of Robot Motion: Theory, Algorithms, and Implementations. Cambridge, MA: MIT Press, 2005.

[2] S. LaValle, Planning Algorithms. New York: Cambridge Univ. Press, 2006.

[3] A. Stentz, "Optimal and efficient path planning for partially-known environments," in Proc. IEEE Int. Conf. Robot. Autom., 1994, pp. 3310-3317.

[4] A. Ettlin and H. Bleuler, "Rough-terrain robot motion planning based on obstacleness," in Proc. Int. Conf. Control, Autom., Robot. Vis., 2006, pp. 1-6.

[5] A. Ettlin and H. Bleuler, "Randomised rough-terrain robot motion planning," in Proc. IEEE/RSJ Int. Conf. Intell. Robots Syst., 2006, pp. 57985803.

[6] C. Urmson and R. Simmons, "Approaches for heuristically biasing RRT growth," in Proc. IEEE/RSJ Int. Conf. Intell. Robots Syst., 2003, pp. 11781183.

[7] D. Ferguson and A. Stentz, "Anytime RRTs," in Proc. IEEE/RSJ Int. Conf. Intell. Robots Syst., 2006, pp. 5369-5375.

[8] R. Diankov and J. Kuffner, "Randomized statistical path planning," in Proc. IEEE/RSJ Int. Conf. Intell. Robots Syst., 2007, pp. 1-6.

[9] J. Kuffner and S. LaValle, "RRT-connect: An efficient approach to singlequery path planning," in Proc. IEEE Int. Conf. Robot. Autom., 2000, pp. $995-1001$.

[10] J. Lee, C. Pippin, and T. Balch, "Cost based planning with RRT in outdoor environments," in Proc. IEEE/RSJ Int. Conf. Intell. Robots Syst., 2008, pp. 684-689.

[11] L. Jaillet, J. Cortés, and T. Siméon, "Transition-based RRT for path planning in continuous cost spaces," in Proc. IEEE/RSJ Int. Conf. Intell. Robots Syst., 2008, pp. 2145-2150.

[12] J.-C. Latombe, Robot Motion Planning. Boston, MA: Kluwer, 1991.

[13] J. Barraquand and J.-C. Latombe, "A Monte-Carlo algorithm for path planning with many degrees of freedom," in Proc. IEEE Int. Conf. Robot. Autom., 1990, pp. 1712-1717.

[14] S. Sekhavat, P. Svestka, and M. Overmars, "Multi-level path planning for nonholonomic robots using semi-holonomic subsystems," Int. J. Robot. Res., vol. 17, no. 8, pp. 840-857, 1998.

[15] R. Geraerts and M. H. Overmars, "Creating high-quality paths for motion planning," Int. J. Robot. Res., vol. 26, pp. 845-863, 2007.

[16] J. Spall, Introduction to Stochastic Search and Optimization. New York: Wiley, 2003

[17] S. Kirkpatrick, C. Gelatt, and M. Vecchi, "Optimization by simulated annealing," Science, vol. 220, pp. 671-680, 1983.

[18] M. Apaydin, A. Singh, D. Brutlag, and J.-C. Latombe, "Capturing molecular energy landscapes with probabilistic conformational roadmaps," in Proc. IEEE Int. Conf. Robot. Autom., 2001, pp. 932-939.

[19] L. Kavraki, P. Svestka, J.-C. Latombe, and M. Overmars, "Probabilistic roadmaps for path planning in high-dimensional configuration spaces," IEEE Trans. Robot. Autom., vol. 12, no. 4, pp. 566-580, Aug. 1996.

[20] S. LaValle, "Rapidly-exploring random trees: A new tool for path planning," Comput. Sci. Dept., Iowa State Univ., Ames, IA, Tech. Rep. TR 98-11, 1998.

[21] A. Yershova, L. Jaillet, T. Siméon, and S. LaValle, "Dynamic-domain RRTs: Efficient exploration by controlling the sampling domain," in Proc. IEEE Int. Conf. Robot. Autom., 2005, pp. 3867-3872.

[22] L. Jaillet, A. Yershova, S. LaValle, and T. Siméon, "Adaptive tuning of the sampling domain for dynamic-domain RRTs," in Proc. IEEE/RSJ Int. Conf. Intell. Robots Syst., 2005, pp. 4086-4091.

[23] T. Siméon, J.-P. Laumond, and F. Lamiraux, "Move3D: A generic platform for path planning," in Proc. IEEE Int. Symp. Assem. Task Plann., 2001, pp. 25-30.

[24] M. Stilman, "Task constrained motion planning in robot joint space," in Proc. IEEE/RSJ Int. Conf. Intell. Robots Syst., 2007, pp. 3074-3081.

[25] J. Cortés, L. Jaillet, and T. Siméon, "Disassembly path planning for complex articulated objects," IEEE Trans. Robot. Autom., vol. 24, no. 2, pp. 475-481, Apr. 2008.

[26] V. Ruiz de Angulo, J. Cortés, and T. Siméon, "BioCD: An efficient algorithm for self-collision and distance computation between highly articulated molecular models," in Robotics: Science and Systems, S. T. and G. Sukhatme, S. Schaal, and O. Brock, Eds. Cambridge, MA: MIT Press, 2005, pp. 6-11.

[27] D. Nieuwenhuisen and M. Overmars, "Useful cycles in probabilistic roadmap graphs," in Proc. IEEE Int. Conf. Robot. Autom., 2004, pp. 446452.

[28] K. Hamacher and W. Wenzel, "Scaling behavior of stochastic minimization algorithms in a perfect funnel landscape," Phys. Rev. E, vol. 59, no. 1, pp. 938-941, Jan. 1999.

[29] D. J. Earl and M. W. Deem, "Parallel tempering: Theory, applications, and new perspectives," Phys. Chem. Chem. Phys., vol. 7, pp. 3910-3916, 2005 .

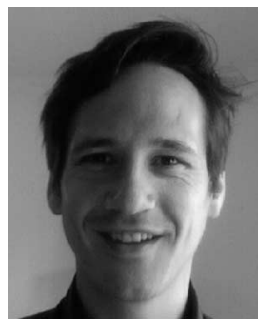

Léonard Jaillet received the engineering degree in mechanical engineering from the Institut Supérieur de Mécanique de Paris, Paris, France, and the Ph.D. degree in robotics from the University of Toulouse, Toulouse, France, in 2001 and 2005, respectively.

Since 2008, he has been a Postdoctoral Fellow with the Institut de Robótica i Informàtica Industrial, Spanish National Research Concil, Barcelona, Spain. His current research interests include motion planning for complex robotic systems and molecular simulations for structural biology.



Juan Cortés received the engineering degree in control and robotics from the Universidad de Zaragoza, Zaragoza, Spain, in 2000 and the Ph.D. degree in robotics from the Institut National Polytechnique de Toulouse, Toulouse, France, in 2003.

Since 2004, he is a Researcher with with the LAAS, Centre National de la Recherche Scientique, Toulouse. His current research interests include the development of algorithms for computing and analyzing the motion of complex systems in robotics and structural biology.

Dr. Cortés is a Co-Chair of the IEEE-RAS TC on Algorithms for Planning and Control of Robot Motion.

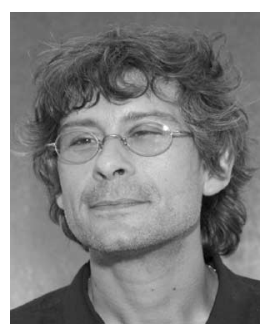

tural bioinformatics.

Dr. Siméon is currently an Associate Editor of the IEEE TRANSACTIONS ON RовотICS.

Thierry Siméon received the engineering degree in computer science from the Institut National des Sciences Appliquées, Toulouse, France, and the Ph.D degree in robotics from the University of Toulouse in 1985 and 1989, respectively.

Since 1990, he has been with the LAAS, Centre National de la Recherche Scientique, Toulouse. $\mathrm{He}$ is the author or coauthor of more than $100 \mathrm{pa}-$ pers. He was a Co-Founder of the LAAS spin-off Kineo Company. His current research interests include robot motion planning and applications to struc- 